\title{
Perfil de idosos atendidos pela Terapia Ocupacional na Residência Multidisciplinar de um hospital público
}

\section{Profile of elderly care in Occupational Therapy in a Multidisciplinary Residency course of public hospital}

\section{Perfil de ancianos atendidos por la Terapia Ocupacional en un curso de Residencia Multidisciplinaria de un hospital público}

Recebido: 26/08/2013

Aprovado: 15/12/2013

\author{
Grasielle Silveira Tavares Paulin 1 \\ Vania Costa Gonçalves da Silva ${ }^{2}$ \\ Anne Marise Koenig3
}

Esta é uma pesquisa quantitativa e descritiva, tendo como objetivo traçar o perfil dos idosos encaminhados ao atendimento em Terapia Ocupacional na Residência Integrada Multiprofissional em Saúde (RIMS) do Hospital de Clínicas em Uberaba. Os resultados evidenciaram que a grande prevalência de múltiplas patologias esteve associada à diminuição da capacidade funcional, à baixa participação social na comunidade, ao impacto das atividades de lazer rotineiras na internação e à correlação existente entre uma boa qualidade de sono e uma melhor capacidade funcional. 0 conhecimento e a análise desses perfis devem facilitar as estratégias de terapia ocupacional para essa população que ganha destaque e necessita de atenção especial.

Descritores: Hospitalização; Idoso; Terapia Ocupacional.

This is a quantitative and descriptive research, aiming to outline the profile of elderly patients referred to the Occupational Therapy service in the RIMS Hospital de Clinicas in Uberaba, Minas Gerais, Brazil. The results showed that the great prevalence of multiple pathologies was associated with reduced functional capacity, low social participation in the community, the impact of leisure activities in routine internment and a good correlation between sleep quality and better functional capacity. The knowledge and analysis of these profiles should facilitate strategies for occupational therapy for this population that stands out and needs special attention.

Descriptors: Hospitalization; Aged; Occupational Therapy.

Esta es una investigación cuantitativa y descriptiva, con el objetivo de trazar el perfil de los ancianos atendidos por el servicio de Terapia Ocupacional en el RIMS del Hospital de Clínicas de Uberaba, Minas Gerais, Brasil. Los resultados mostraron que la gran prevalencia de patologías múltiples se asoció con la disminución de la capacidad funcional, la baja participación social en la comunidad, el impacto de las actividades de ocio en la internación de rutina y una buena correlación entre la calidad del sueño y una mejor capacidad funcional. El conocimiento y análisis de estos perfiles debe facilitar las estrategias de la terapia ocupacional para esta población que se destaca y necesita de atención especial.

Descriptores: Adaptación, Anciano, Hogares para Ancianos.

\footnotetext{
1 Terapeuta Ocupacional. Doutora em Saúde Pública. Professora Adjunta do Departamento de Terapia Ocupacional da Universidade Federal do Triângulo Mineiro (UFTM). grasiellet@yahoo.com.br

2 Terapeuta Ocupacional. Especialista pela Residência Integrada Multiprofissional em Saúde Área de Concentração Saúde do Idoso-UFTM. vaniacgsilva@hotmail.com

3 Professora Assistente do Departamento de Terapia Ocupacional-UFTM. annekoenig.to@gmail.com
} 


\section{INTRODUÇÃO}

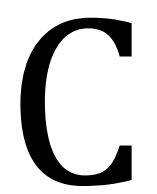

om o perfil demográfico da população em franca transição, observam-se mudanças que acarretam uma série de consequências sociais, culturais e epidemiológicas, além do impacto no setor saúde, em especial, o hospitalar. Muitos estudos têm demonstrado preocupação em conhecer o perfil de hospitalização da população idosa. Em geral, esse perfil aponta para condições de saúde, aspectos socioeconômicos, doenças crônicas e declínio funcional ${ }^{1,2}$.

As doenças nos idosos exigem cuidados específicos das equipes multiprofissionais. Por serem crônicas e múltiplas, acabam consumindo mais serviços de saúde do que pessoas de outras faixas etárias. As internações hospitalares são frequentes e o tempo de ocupação do leito é maior ${ }^{3}$.

0 indivíduo que passa por um período de hospitalização enfrenta alterações consideráveis no seu dia a dia. Ao ser inserido no contexto hospitalar, vivencia a falta do ambiente familiar, pode ficar exposto em períodos de realização de exames, expectativas de diagnósticos e dor, vivenciando desconforto físico, moral e espiritual4. Durante esse período, o idoso tem poder mínimo sobre suas ações e o declínio funcional pode ser comprometido, levando-o à insegurança em virtude da dependência, ansiedade, angústia e até mesmo o medo da morte 5 .

Os profissionais da área de saúde necessitam entender que as práticas realizadas devem ser destaque para a dimensão da humanização e da integralidade do cuidado, conforme proposta pelo Sistema Único de Saúde (SUS).

A Residência Integrada Multiprofissional em Saúde da Universidade Federal do Triângulo Mineiro (RIMS-UFTM), área de concentração Saúde do Idoso, surgiu com uma abordagem voltada para a atuação em equipe multiprofissional, com atenção integral e humanizada em saúde. A abordagem do terapeuta ocupacional ganha ênfase nesse contexto, visto que as áreas de ocupação do idoso são bastante afetadas, principalmente com a hospitalização.

0 objetivo deste trabalho foi o de traçar o perfil dos idosos internados na Clínica Médica do Hospital de Clínicas da UFTM que foram encaminhados para a Terapia Ocupacional.

\section{MÉTODO}

Esta é uma pesquisa quantitativa, documental, retrospectiva e descritiva, e que visa fornecer dados e conhecimento para delineamento de ações futuras com os idosos a serem atendidos.

O local de estudo foi o Hospital de Clínicas da UFTM (HC-UFTM), que assiste a população de Uberaba e região, compreendendo 27 municípios que compõem a macrorregião do Triângulo Sul. Como único hospital público que oferece atendimento terceirizado de alta complexidade, abrange também outras regiões de Minas Gerais e outros estados da federação.

A amostra compreendeu idosos atendidos na Clínica Médica do HC, com idade igual ou superior a 60 anos, de ambos os sexos, residentes em Uberaba e região. Essa população foi assistida pela equipe da RIMS, na área de concentração Saúde do Idoso, e encaminhada ao atendimento de terapia ocupacional no período de internação de maio a dezembro de 2010, tendo assinado o Termo de Consentimento Livre e Esclarecido (TCLE).

Para a coleta dos dados, foi utilizado um instrumento denominado histórico de saúde. Esse documento foi criado $\mathrm{e}$ elaborado por cada área de concentração da RIMS e objetivava conhecer e identificar a realidade da população e os problemas relacionados aos aspectos psicossociais, econômicos, às disfunções físicas e cognitivas. Posteriormente, pretendia proporcionar a discussão de casos, a identificação das demandas e definição de 
plano de intervenção à cada área profissional.

O instrumento é constituído por 20 domínios, sendo eles: Identificação, Situação ocupacional, Situação sócio familiar, Situação habitacional, Diagnóstico médico, História pregressa e atual, Medicação, Hábitos de consumo, Cognição, Atividade sexual, Sono e repouso, Exercício físico, Histórico familiar, Mobilidade, Atividades de Vida Diária (AVDs), Atividade Instrumental de Vida Diária (AIVD), Lazer, Avaliação de saúde, Redes sociais e Queixa principal.

Após a aprovação do estudo pelo Comitê de Ética em Pesquisa sob o n. 1819 , realizado de acordo com as normas da Resolução 196/96 do Conselho Nacional de Ética e Pesquisa, definiu-se a amostra selecionando os históricos de saúde e respeitando os critérios de inclusão já citados. Participaram da pesquisa 52 sujeitos, que responderam ao histórico de saúde e assinaram o TCLE.

Os dados coletados foram armazenados e tabulados no software Microsoft Excel 2007 e analisados por meio de frequência simples. Como a quantidade de dados obtidos foi extensa, optou-se por descrever e compreender a relação entre as variáveis sociodemográficas, $\quad 0$ desempenho ocupacional e quadros clínicos prevalentes visando atender ao objetivo do estudo.

\section{RESULTADOS}

\section{Aspectos sociodemográficos}

Os resultados relacionados à caracterização do perfil demográfico apontam que a idade variou de 60 a 95 anos, com média de 73 anos, sendo 58\% dos idosos do sexo masculino e $42 \%$ residentes em outras cidades atendidas pelo HC/UFTM. No que se refere ao estado civil, $48 \%$ estão casados, prevalecendo o sexo masculino nesta condição.

Quanto à escolaridade, 35\% dos sujeitos são analfabetos e $52 \%$ não concluíram o ensino fundamental. A religião de $60 \%$ é a católica; $17 \%$ evangélicos; $2 \%$ espíritas; $19 \%$ não se manifestaram quanto à sua religião; e $2 \%$ não praticam religião, porém afirmam espiritualidade.

Sobre a renda mensal, notou-se que $75 \%$ recebem até dois salários mínimos (SM); $13 \%$, até três; $6 \%$ contam de quatro até seis $\mathrm{SM}$; 4\% não dispõem de nenhuma renda; e $2 \%$ não informaram sua renda. Observou-se que os idosos, em sua maioria, são os provedores de seus lares.

\section{Função/Desempenho Ocupacional}

$\mathrm{Na}$ avaliação do desempenho ocupacional para realizar as Atividades de Vida Diária AVDs, o instrumento avaliou as seguintes variáveis: banho, controle de esfíncter, vestimenta, alimentação, locomoção e higiene pessoal. Para as Atividades Instrumentais de Vida Diária - AIVDs considerou-se: preparar as refeições, fazer o trabalho doméstico, lembrar-se de tomar os remédios, cuidar das próprias finanças, fazer compras e usar transporte coletivo.

$\mathrm{Na}$ Tabela 1, está representado o resultado do Desempenho Ocupacional na realização das AVDs, em que se observou o predomínio de $80 \%$ dos idosos que desempenham sem ajuda a maioria dessas atividades. Notou-se que $13 \%$ são parcialmente dependentes, ou seja, necessitam da ajuda de outra pessoa ou de um equipamento de tecnologia assistiva para realizar algumas das atividades, e $5 \%$ necessitam de ajuda total, estando acamados e dependentes.

Tabela 1. Desempenho Ocupacional nas AVDs e AIVDs. HC/UFTM-Uberaba, 2010.

\begin{tabular}{lllcc|}
\hline & $\begin{array}{l}\text { Sem } \\
\text { ajuda }\end{array}$ & $\begin{array}{l}\text { Ajuda } \\
\text { Parcial }\end{array}$ & $\begin{array}{l}\text { Ajuda } \\
\text { Total }\end{array}$ & $\begin{array}{l}\text { Não } \\
\text { Informado }\end{array}$ \\
\hline AVDs & $80 \%$ & $13 \%$ & $5 \%$ & $2 \%$ \\
\hline AIVDs & $60 \%$ & $15 \%$ & $23 \%$ & $2 \%$ \\
\hline
\end{tabular}

Dos idosos do sexo masculino que necessitam de ajuda parcial, 35\% têm, no máximo, 70 anos e muitas patologias prevalentes, como se pode observar no Gráfico 2 e na Tabela 2, com associação entre essas duas variáveis. Nas mulheres, $43 \%$ declararam necessitar de ajuda total e 
estão na mesma faixa etária dos homens, como representado no Gráfico 1.

Gráfico 1. AVDs em Idosas. HC-UFTM, 2010.

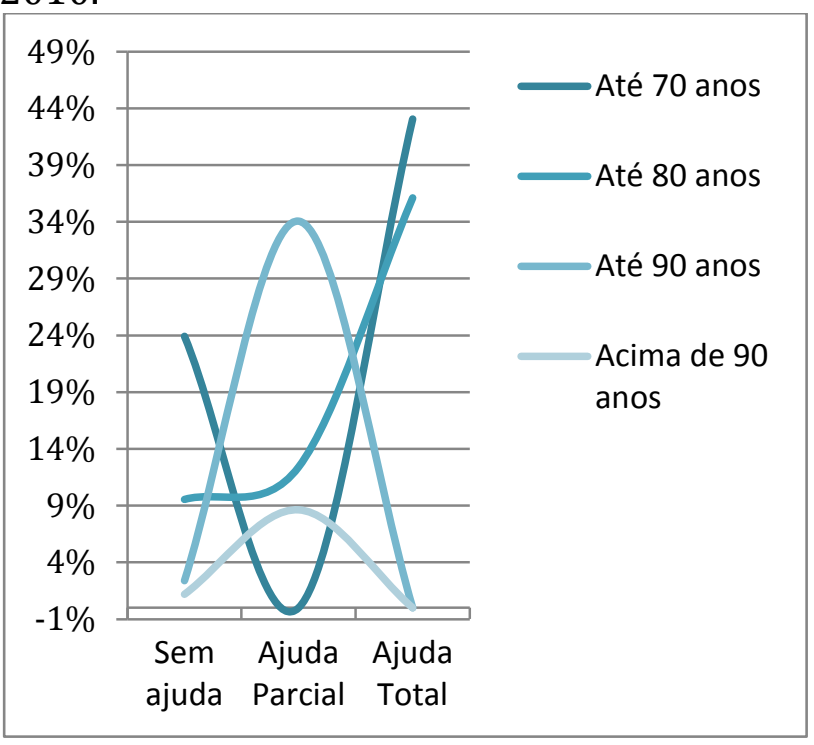

Gráfico 2. AVDs em Idosos. HC-UFTM, 2010.

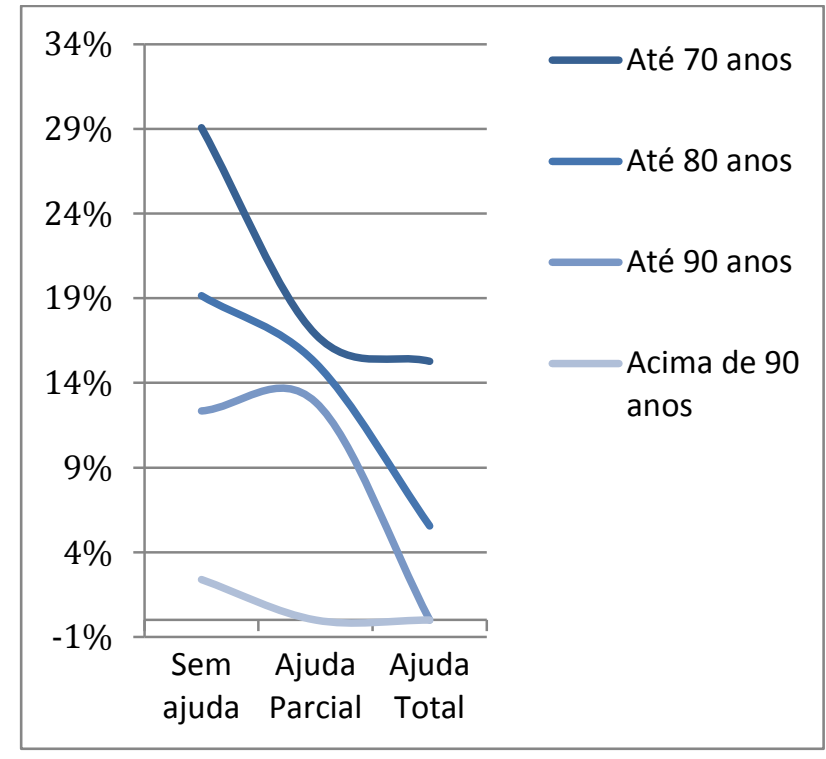

Em relação ao Desempenho Ocupacional das AIVDs, observa-se, na Tabela 1, que $40 \%$ desses idosos apresentaram declínio no desempenho de algumas atividades, como: preparar as refeições, cuidar das finanças e fazer compras, sendo estes principalmente do sexo feminino com até 70 anos, enquanto $23 \%$ necessitam de ajuda total para desempenhar algumas dessas atividades, conforme mostra o Gráfico 3. Nesse subgrupo, classificam-se em sua maioria também mulheres, porém em outra faixa etária, até 90 anos.

Gráfico 3. AIVDs em Idosas. HC, 2010.

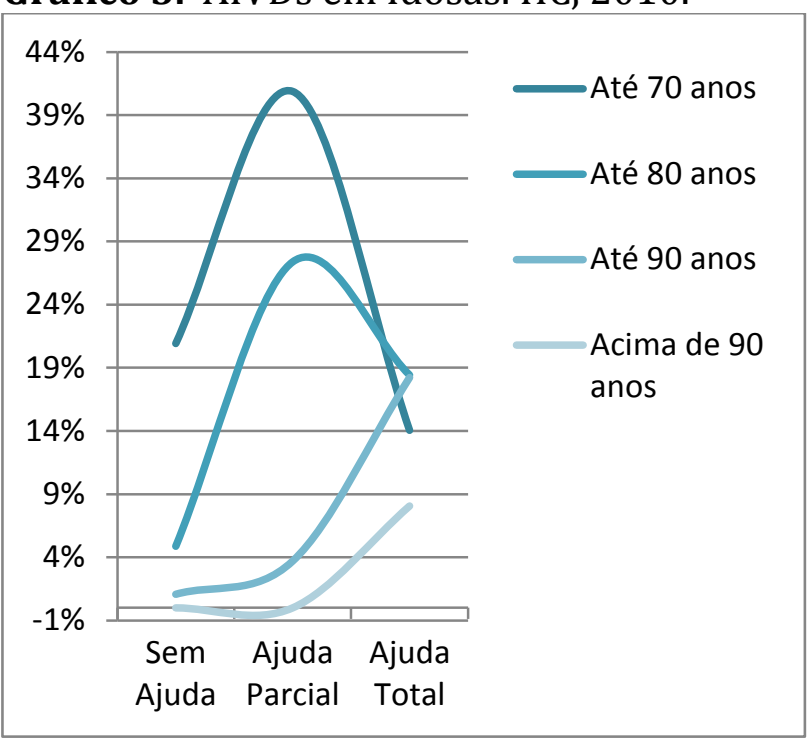

Gráfico 4. AIVDs em Idosos. HC, 2010.

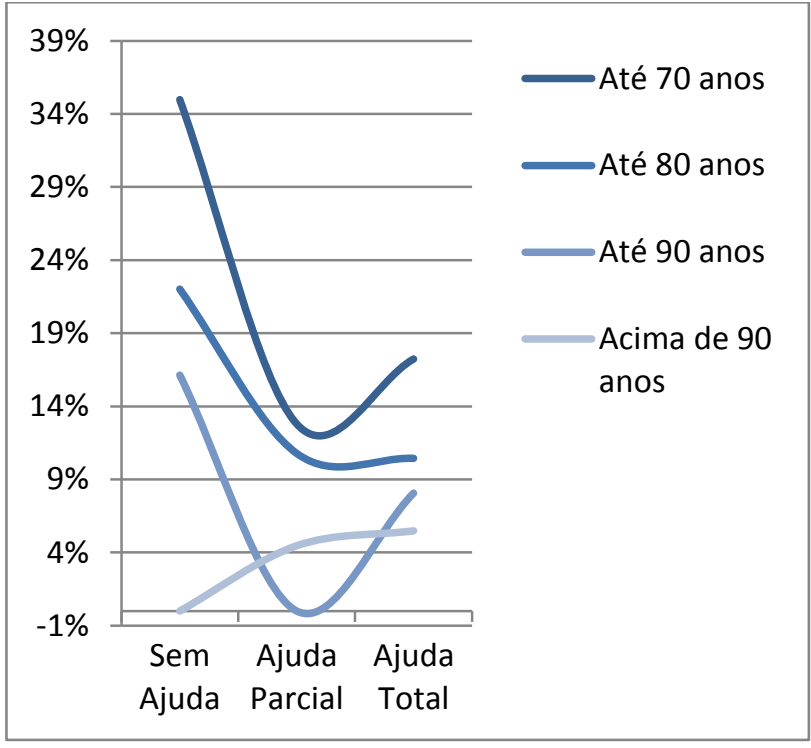

Quanto ao sono e ao repouso, os dados estão representados nos Gráficos 5 e 6, demonstrando que $17 \%$ consideram o sono ruim e $20 \%$ tomam medicação para dormir. Aqueles que declararam dormir mal coincidem com o grupo que apresenta as AVDs e AIVDs mais prejudicadas, ou seja, em sua maioria, $67 \%$ são mulheres distribuídas em duas faixas etárias: até 70 anos ou até 90 anos, com maior predominância da segunda faixa etária. 
Gráfico 5. Sono e Repouso/AVDs. HC, 2010.

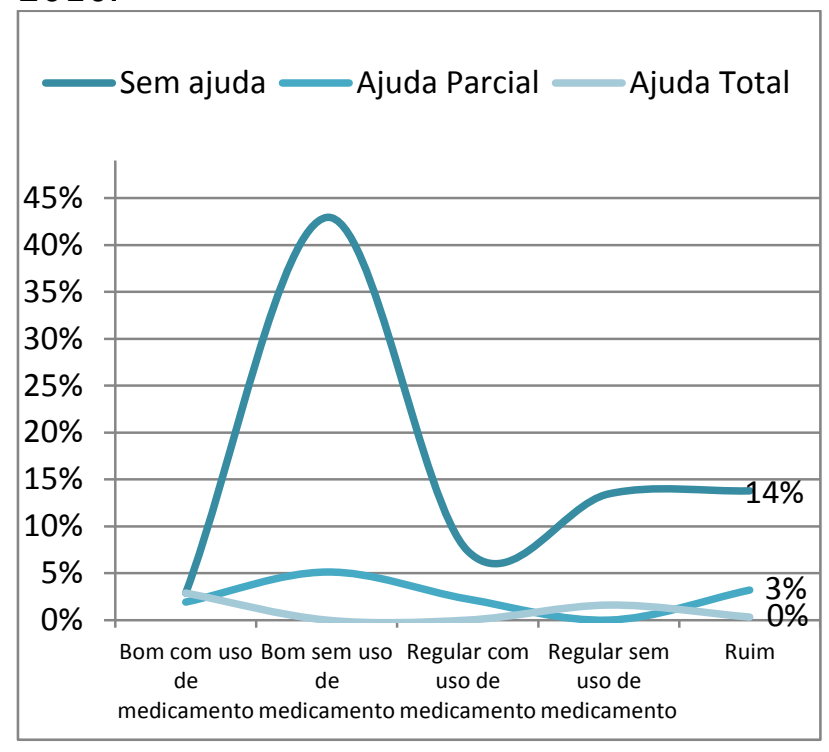

Gráfico 6. Sono e Repouso/AIVDs. HC, 2010.

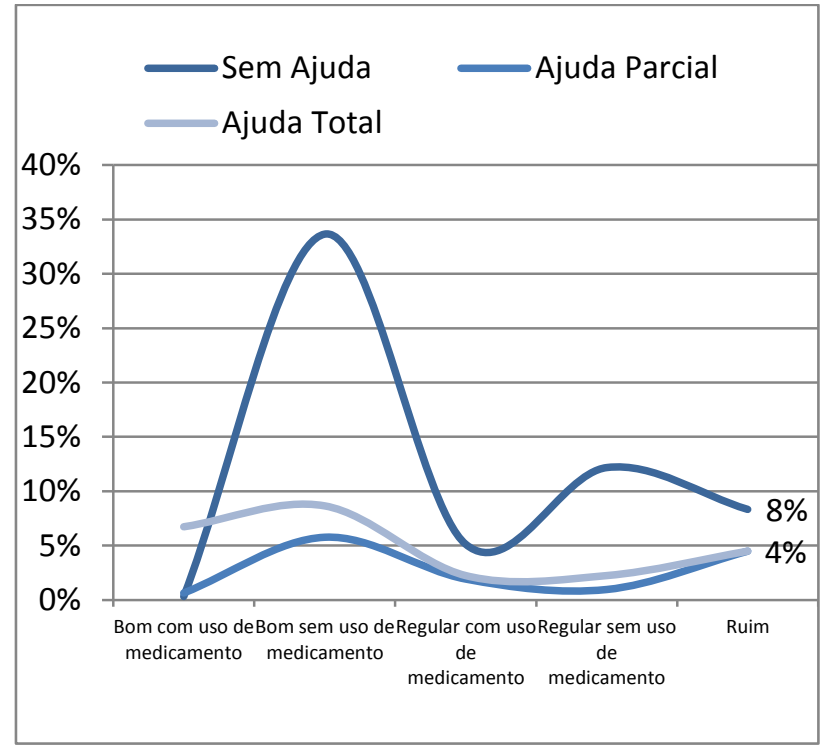

Em relação à participação social, 58\% não participam de nenhuma atividade. Apenas três idosos referiram frequentar locais que oferecem atividades como bailes para a terceira idade, e dois participam de campeonatos de truco. Os demais consideraram como participação social frequentar a igreja e participar de grupos de oração. Em relação ao lazer, as atividades citadas pelos idosos foram: $67 \%$ - televisão; 38\% - rádio; 17\%, - ouvir músicas e dançar; $10 \%$ - pescar; $4 \%$ leitura; e 13\% praticam algum tipo de jogo.

\section{Quadros clínicos prevalentes}

Quanto às principais causas de internação hospitalar em idosos, na Tabela 2 há prevalência das doenças de acordo com sexo, notando-se que as mais frequentes relacionam-se às patologias do sistema cardiovascular (33\%) e endócrino (23\%), seguidas das neurológicas e pneumológicas.

Tabela 2. Quadros Clínicos Prevalentes por área e gênero. HC/UFTM-Uberaba, 2010.

\begin{tabular}{lrrrrrr} 
& \multicolumn{1}{c}{ Feminino } & Masculino & \multicolumn{2}{c}{ Total } \\
\hline Cardiologia & 18 & $45 \%$ & 22 & $55 \%$ & 40 & $33 \%$ \\
\hline Endocrinologia & 16 & $57 \%$ & 12 & $43 \%$ & 28 & $23 \%$ \\
\hline Neurologia & 4 & $29 \%$ & 10 & $71 \%$ & 14 & $12 \%$ \\
\hline Pneumologia & 6 & $50 \%$ & 6 & $50 \%$ & 12 & $10 \%$ \\
\hline Urologia & 5 & $50 \%$ & 5 & $50 \%$ & 10 & $8 \%$ \\
\hline Ortopedia & 3 & $50 \%$ & 3 & $50 \%$ & 6 & $5 \%$ \\
\hline Oncologia & 0 & $0 \%$ & 2 & $100 \%$ & 2 & $2 \%$ \\
\hline Outras & 4 & $50 \%$ & 4 & $50 \%$ & 8 & $7 \%$ \\
\hline
\end{tabular}

Verificou-se que dois pacientes na faixa etária de 70 anos apresentaram sete patologias e $84,61 \%$ já possuíam histórico de internações anteriores. 0 período de internação prevalente é de até 10 dias para pacientes com faixa etária de até 70 anos. Esses mesmos pacientes apresentaram múltiplas patologias.

\section{DISCUSSÃO}

Os dados relacionados à caracterização do perfil demográfico corroboram as informações obtidas por meio do Sistema de Informações Hospitalares do Sistema Único de Saúde, SUS - SIH/SUS, DATASUS 6 no município de Uberaba-MG em 2010, onde o maior número de internações ocorreu na faixa etária dos 60 a 69 anos, seguida dos que estavam na faixa etária de 70 a 79 anos. Verificou-se um predomínio do sexo masculino em 58\%. A maioria dos indicadores de saúde mostra existência de diferencial de saúde entre gêneros e tem estudado a razão pela qual os homens são mais vulneráveis às doenças e morrem mais precocemente que as mulheres.

A vida média masculina é comparativamente menor, as mulheres têm maior sobrevida. Apesar de apresentarem 
maior incidência de episódios agudos de doenças, as mulheres se cuidam mais ou aderem a programas de prevenção e tratamentos, diferentemente dos homens, que se cuidam menos e fazem menos exames preventivos, tornando-se as principais vítimas de doenças crônicas e de outros problemas de saúde que podem levar à morte ${ }^{7}$.

No que se refere ao estado civil, prevaleceram os que estão casados e são do sexo masculino, enquanto, entre viúvos, predominou o sexo feminino. A situação da viuvez para o sexo feminino ocorre, em especial, por causa de sua alta expectativa de vida, sendo que, ao se tornarem viúvas, permanecem nessa situação. Por sua vez, ao perderem as parceiras, os homens têm a tendência a se casarem novamente ${ }^{8}$.

Quanto à escolaridade, observou-se que em maior número estão os que não atingiram o ensino médio e possuem pouca instrução, seguidos dos analfabetos. A escolaridade é um dado a ser valorizado, pois a falta de comunicação e entendimento interfere nas reações e no comportamento da pessoa que está em processo de doença e hospitalização.

Ao passar pelo processo de internação, o idoso vivencia um desconforto, ficando exposto ao medo do desconhecido e a procedimentos muitas vezes invasivos. Nas orientações sobre a condição de saúde, a comunicação com os idosos se faz em uma linguagem técnica, o que aumenta sua preocupação e ansiedade 9 . Esses dados indicam a necessidade dos profissionais de transmitirem informações ao idoso e à sua família ou cuidador com um vocabulário simples, que faça sentido para que essas orientações possam ser colocadas em prática no cotidiano.

As necessidades espirituais dos idosos devem ser consideradas e facilitadas pelo serviço de saúde no período de internação. De acordo com a American Occupational Therapy Association (AOTA) ${ }^{10}$, a espiritualidade é um dos fatores do cliente que influencia e motiva o indivíduo a se envolver em ocupações e fornece significado e qualidade de vida. A abordagem espiritual é uma estratégia de enfrentamento da doença, uma forma de compreender o processo de morte e de se sentir paz.

A maioria dos idosos dispõe de um a dois SM, sendo, a maior parte destes, os únicos provedores de seus lares. Nesse contexto, a aposentadoria passa a ser a única fonte de renda e não preenche suas necessidades básicas, como saúde, alimentação, moradia e até mesmo lazer ${ }^{11}$. 0 fato se agrava quando se considera que esses mesmos aposentados necessitam comprar os remédios essenciais para seus cuidados. Apesar de alguns medicamentos estarem disponíveis gratuitamente na rede pública, $40 \%$ dos entrevistados declararam serem os mantenedores de sua medicação.

No perfil de Desempenho Ocupacional, o referencial teórico que fundamentou o trabalho foi o documento da AOTA ${ }^{10}$, sendo o desempenho ocupacional a habilidade para seguir e manter uma rotina diária, executar as atividades de autocuidado, sono, desempenhar papéis sociais e desfrutar do lazer de modo satisfatório e apropriado para a condição de saúde, idade e cultura no contexto no qual se está inserido. Neste estudo foram consideradas cinco áreas de ocupação (AVDs, AIVDs, sono, participação social e lazer) que constavam no histórico de saúde.

É sabido que as perdas funcionais ocorrem quase duas vezes mais para as AIVDs do que para as AVDs. Portanto, idosos que apresentam algum comprometimento na habilidade para desempenhar alguma das AVDs provavelmente já apresentam também inabilidade para duas ou mais das AIVDs. Isso significa que, em geral, a incapacidade nas AIVDs precede 0 comprometimento das atividades de autocuidado, sendo um indicativo da necessidade de ações de prevenção de agravos e promoção da saúde ${ }^{12}$.

A funcionalidade e a capacidade de desempenhar as atividades do cotidiano são influenciadas pelo processo de envelhecimento fisiológico, por 
características de gênero, classe social, renda, escolaridade, condições de saúde, cognição, ambiente e história de vida13. Além disso, os efeitos da hospitalização, somados a condições clínicas prévias, independente do ciclo vital, dos motivos e do tempo de internação, acarretam alterações biopsicossociais na vida da pessoa que adoece. Isso leva os indivíduos internados, a experimentarem o impacto funcional e a suspensão temporária ou permanente da independência em atividades de vida diária ${ }^{14}$. A dificuldade ou comprometimento que incapacita a realização individual das atividades diárias limita o idoso, reduz sua qualidade de vida e aumenta o risco de dependência, institucionalização e morte prematura.

Os que declararam dormir mal, também apresentam as AVDs e AIVDs mais prejudicadas. 0 sono é uma manifestação biológica, regulador essencial das condições de saúde e grande responsável pelo desempenho físico e mental de uma pessoa e, por isso, é capaz de influenciar na coordenação motora, na ansiedade, na disposição emocional e no desempenho cognitivo ${ }^{15}$.

Dos idosos pesquisados, $58 \%$ não participam de nenhuma atividade. Os demais consideraram como participação social frequentar a igreja e se inserirem em grupos de orações. Dentre os critérios descritos pela AOTA $^{10}$, quando se fala em participação social, são levados em consideração padrões organizados de comportamentos que são característicos e esperados de um indivíduo ou de determinada posição no sistema social. Estes envolvem atividades que resultem em interações que influenciam na participação nos espaços e nas organizações da comunidade e da sociedade, enquanto o lazer pode ser visto como cultura compreendida em seu sentido mais amplo, vivenciada no tempo disponível. Assim, qualquer atividade prazerosa pode ser considerada de lazer ${ }^{16}$.

As limitações de lazer apresentadas pelo indivíduo durante a hospitalização, a restrição de atividades e da participação social, além dos fatores ambientais e pessoais, interferem no desempenho das AVDs e podem funcionar como barreiras do estado funcional. A classe social, o nível de instrução, a faixa etária, o gênero, e outros fatores, limitam as oportunidades de prática do lazer e são indicadores indesejáveis que necessitam ser reavaliados por uma política que objetive a democratização do lazer ${ }^{16}$.

As doenças crônicas são as principais causas de incapacidade para os idosos, além de serem responsáveis por elevado número de óbitos e de mortes prematuras. Quanto às principais causas de internação hospitalar em idosos, as patologias do sistema cardiovascular e endócrino, seguidas das neurológicas e pneumológicas foram as de maior predomínio. Esse resultado coincide com outros achados, afirmando que cerca de metade das internações hospitalares de idosos tem como causas mais frequentes afecções cardíacas e respiratórias ${ }^{17}$.

A elevada prevalência de doenças crônico-degenerativas, somada à ocorrência de duas ou mais doenças concomitantes, tem sido considerada responsável pela necessidade de maior permanência hospitalar e pela progressiva perda de autonomia dos idosos.

\section{CONCLUSÃO}

No perfil de idosos encaminhados para a intervenção de Terapia Ocupacional observou-se que a maioria é do sexo masculino, casado, com a média de idade de 73 anos, prevalência de múltiplas patologias, e necessitando de ajuda parcial em determinadas atividades. Em relação às mulheres, a maioria está viúva, com a mesma média de idade e com menor número de patologias, porém com maior grau de comprometimento.

É necessário na saúde do homem desenvolver-se estratégias para a prevenção de doenças e promoção da saúde nos serviços de atenção primária. Isso proporciona a participação dos idosos de forma ativa na sociedade por meio de 
atividades significativas, que contribuirão para a melhora da qualidade de vida, podendo reduzir as hospitalizações. Ambos os sexos apresentaram baixo grau de escolaridade e de renda, fatores que podem dificultar a comunicação, o autoentendimento e o gerenciamento dos problemas pelo idoso. Esses dados indicam a necessidade dos profissionais de transmitirem informações ao idoso e à sua família ou ao seu cuidador usando vocabulário simples, que faça sentido para que estas possam ser colocadas em prática no cotidiano.

Dos pesquisados, a maioria já teve internações anteriores, com baixa participação social na comunidade, interrupção nas atividades de lazer rotineiras durante a hospitalização e correlação existente entre uma boa qualidade de sono e melhor capacidade funcional.

É necessário que os profissionais tenham visão além da doença, de seus sintomas e limitações impostas, que valorizem a bagagem e a experiência de vida do idoso. É importante que o cuidado seja voltado para o lazer, valorizando a espiritualidade e a integralidade do cliente que passa pelo processo de hospitalização, ficando exposto à dor, ao medo e a outras dificuldades.

Esse novo olhar para a população idosa é de grande valia nas ações de Terapia Ocupacional no contexto hospitalar.

Sugere-se que novos estudos possam ser realizados para identificar as necessidades ou problemas que afetam os idosos durante o processo de hospitalização.

\section{REFERÊNCIAS}

1. Sales FM, Santos I. Perfil de idosos hospitalizados e nível de dependência de enfermagem: identificação de necessidades. Texto e Contexto Enferm. 2007; 16(3):495-502.

2. Sthal HC, Berti HW, Palhares VC. Grau de Dependência de idosos hospitalares para realização das atividades básicas da vida diária. Texto e Contexto Enferm. 2011; 20(1):59-67.

3. Pavarani SCI, Mendiondo MSZ, Barham EJ, Veroto VAG, Filizola CLA. A arte de cuidar do idoso: gerontologia como profissão?.Texto e Contexto Enferm. [Internet]. 2005; 14(3):398-402. [acesso em 20 jan 2011]. Disponível em: http://www.scielo.br/pdf/tce/v14n3/v14 n3a11.pdf

4. Soares NN, Custódio MRM. Impactos emocionais da alteração da rotina em idosos hospitalizados. Encontro [Internet]. 2011; 14(21):9-23. [acesso em 20 nov 2011]. Disponível em: http://sare.anhanguera.com/index.php/re ncp/issue/view/94

5. Dalbosco SNP. O idoso hospitalizado: perspectivas do próprio sujeito a respeito de si mesmo, dos familiares e dos profissionais cuidadores [Internet]. [dissertação]. Porto Alegre: Faculdade de Educação do Rio Grande do Sul, Universidade Federal do Rio Grande do Sul; 2009. [acesso em 20 nov 2011]. Disponível em:

http://www.lume.ufrgs.br/bitstream/hand le/10183/18267/000727764.pdf?sequenc $\mathrm{e}=1$

6. Ministério da Saúde (Br). DATASUS: Informações de Saúde: indicadores e dados básicos - IDB - 2010 [Internet]. Brasília: Ministério da Saúde; [2010] [acesso em 10 jan 2012]. Disponível em: http://www2.datasus.gov.br/DATASUS/in dex.php/index.php?acao $=11 \& i d=29010$

7. Laurenti R, Jorge MHPM, Gotlieb SLD. Perfil epidemiológico da morbimortalidade masculina. Ciênc Saúde Coletiva 2005; 10(1):35-45.

8. Camarano AA. Mulher Idosa: suporte familiar ou agente de mudança? Estud Av. 2003; 17(49):35-63.

9. Oriá MOB, Moraes LMP, Victor JF. A comunicação como instrumento do enfermeiro para o cuidado emocional do cliente hospitalizado. Rev Eletrônica Enferm. [Internet]. 2004; 6(2):292-7. [acesso em 20 nov 2011]. Disponível em: 
http://www.revistas.ufg.br/index.php/fen /article/view/808/922.

10. The American Occupational Therapy Association. Estrutura da prática da terapia ocupacional: domínio e processo: 2 ed . Carleto DGS, Souza ACA, Silva M, Cruz DMC, Andrade VS, tradutores. Rev Triang.: Ens Pesq Ext. [Internet]. 2010; 3(2):57-147. [acesso em 20 jan 2011]. Disponível em: http://www.uftm.edu.br/revistaeletronica /index.php/revistatriangulo/article/view/ $150 / 177$

11. Freitas DG. Idoso provedor de família [Internet]. [Monografia]. Presidente Prudente: Faculdade de Serviço Social de Presidente Prudente, Faculdades Integradas Antônio Eufrásio de Toledo; 2005 [acesso em 20 nov 2011]. Disponível em:

http://intertemas.unitoledo.br/revista/ind ex.php/Juridica/article/viewFile/400/395

12. Pedrazzi EC, Rodrigues RAP, Schiaveto FV. Morbidade referida e capacidade funcional de idosos. Ciênc Cuid Saúde 2007; 6(4):407-13.

13. Perracini MR, Fló CM. Funcionalidade e envelhecimento. Rio de Janeiro: Guanabara Koogan; 2009.

14. Rafani SM, Santana S, Prado KCG. O cuidado ao individuo idoso em contexto de internação: a intervenção da terapia ocupacional na enfermaria de geriatria do HC/FMRP-USP. In: Uchoa-Figueiredo LR, Negrini SBM. Terapia ocupacional: diferentes práticas em hospital geral. Ribeirão Preto: Legis Summa; 2009. p. 27988.

15. Leite FAP, Soler VM. Sono e Repouso: realidade de uma população de idosos hospitalizados. CuidArt Enferm. [Internet]. 2008; 2(2):134-43. [acesso em 20 nov 2011]. Disponível em: http://www.fundacaopadrealbino.org.br/f acfipa/ner/pdf/ed03enfpsite.pdf

16. Marcellino NC. Estudos do Lazer: uma introdução. 4 ed. Campinas: Autores Associados; 2006.

17. Siqueira AB, Cordeiro RC, Perracini MR, Ramos R. Impacto funcional da internação hospitalar de pacientes idosos. Rev Saúde Pública 2004; 35(5):687-94.

\section{CONTRIBUIÇÕES}

Grasielle Silveira Tavares Paulin participou na concepção, delineamento, análise e interpretação dos dados e redação;

Vania Costa Goncalves da Silva atuou na concepção, delineamento, análise e interpretação dos dados e redação;

Anne Marise Koenig contribuiu na análise e interpretação dos dados, redação e revisão crítica. 